\title{
Un disciple militant et son maître : Charles Baudouin et Romain Rolland
}

\section{Antoinette Blum}

\section{(2) OpenEdition}

\section{Journals}

Édition électronique

URL : http://journals.openedition.org/edl/340

DOI : $10.4000 /$ edl.340

ISSN : 2296-5084

Éditeur

Université de Lausanne

\section{Édition imprimée}

Date de publication : 15 septembre 2012

Pagination : 49-74

ISBN : 978-2-940331-28-4

ISSN : 0014-2026

\section{Référence électronique}

Antoinette Blum, "Un disciple militant et son maître : Charles Baudouin et Romain Rolland », Études de lettres [En ligne], 3 | 2012, mis en ligne le 15 septembre 2015, consulté le 19 décembre 2020. URL :

http://journals.openedition.org/edl/340 ; DOI : https://doi.org/10.4000/edl.340 


\section{UN DISCIPLE MILITANT ET SON MAÎTRE: CHARLES BAUDOUIN ET ROMAIN ROLLAND}

Le psychanalyste et écrivain Charles Baudouin fut un des nombreux disciples de Romain Rolland. L'écrivain "fut peut-être, par son appel "Au-dessus de la mêlée" la raison la plus déterminante de [1] a venue en Suisse» en 1915 du jeune Français, réformé de l'armée. Pendant près de trente ans, Baudouin entretient des liens étroits avec Rolland, même lorsqu'il se sépare de lui sur le plan politique pendant les années 30. Des extraits du journal en grande partie toujours inédit de Baudouin - le Carnet de route révèlent non seulement l'admiration que Baudouin porte à l'écrivain, mais également la fascination qu'il exerce sur lui. Baudouin voudra donc rendre hommage à Rolland et à son œuvre de façons diverses jusqu'à la mort de ce dernier en 1944: il publie des articles de Rolland dans sa revue Le Carmel (1916-1918), il rédige ses propres textes sur l'écrivain et il tente de mettre sur pied des hommages collectifs célébrant Rolland. C'est ainsi que Baudouin put exprimer toute sa reconnaissance envers un de "ses pères vivants».

Le lien intellectuel, littéraire et affectif entre Charles Baudouin et Romain Rolland a déjà fait l'objet de plusieurs études ${ }^{1}$. Nous nous proposons cependant ici d'analyser leur rapport selon une perspective plus restreinte. Notre regard portera avant tout sur la tentative du psychanalyste, écrivain, poète, critique et traducteur Charles Baudouin (18931963) de rendre hommage à Romain Rolland (1866-1944) et à son œuvre de façons diverses: il a publié des articles de Rolland, a rédigé ses propres textes sur l'écrivain et tenté de mettre sur pied des hommages

I. Pour cet article, je me suis inspirée de mes travaux déjà publiés sur Baudouin. Voir A. Blum (éd.), Correspondance entre Romain Rolland et Charles Baudouin; Charles Baudouin, Romain Rolland; "Regard sur la Correspondance (1916-1944) entre Romain Rolland et Charles Baudouin». 
collectifs célébrant Rolland. Seront cités également ici des passages du journal de Baudouin - son Carnet de route - où le jeune disciple évoque ses rencontres avec Rolland et nous livre au long des années ses réflexions sur son maitre, autant d'hommages mais de caractère privé qu'il consacre à une figure paternelle, à un de ses "pères vivants" ${ }^{2}$.

Jeune Français de vingt-deux ans, mobilisé en 1914, mais très vite réformé en raison de sa santé fragile, Baudouin décide en 1915 de se rendre en Suisse et de s'installer à Genève. C'est dans cette même ville qu'il meurt en 1963. Trois raisons avaient dicté son choix de cette ville: l'état de ses poumons, son désir d'enseigner dans l'Institut JeanJacques Rousseau récemment fondé et son espoir d'y rencontrer Romain Rolland, "l'homme qui fut peut-être, par son appel "Au-dessus de la mêlée", la raison la plus déterminante de ma venue en Suisse» " ${ }^{3}$ écrit Baudouin dans son Carnet de route. Il avait découvert à Paris en août 1915 l'article détonateur de l'écrivain, paru à l'origine dans le Journal de Genève le 22 septembre 1914, mais reproduit dans le pamphlet du nationaliste français Henri Massis, Romain Rolland contre la France.

\section{Autour de Carl Spitteler et de Romain Rolland}

Baudouin rend visite à Rolland dix jours après son arrivée à Genève. Mais même avant son premier entretien avec l'écrivain, il fait une rencontre qui, écrit Baudouin, "pourrait bien marquer une date décisive dans ma vie» et qu'il attribue "au Destin ou à la Providence " ${ }^{4}$, c'est celle du grand poète suisse-allemand, Carl Spitteler, auteur des épopées Prométhée et Epiméthée et Le Printemps Olympien, avec qui Rolland entretenait des relations depuis avril 1915. Apprenant de façon fortuite que Spitteler se trouvait dans l'atelier du sculpteur James Vibert à Chapelle-sur-Carouge qui - pour fêter le jubilée de ses soixantedix ans - s'était mis à sculpter son buste, Baudouin s'y rend. Il décrit

2. Ch. Baudouin, "Textes autobiographiques: "Connais-toi toi-même" ", f. 5. Il avait perdu son propre père à l'âge de onze ans.

3. Ch. Baudouin, Carnet de route, "Au-dessus de la mêlée», 25 octobre 1915, f. 30. Plusieurs passages du Carnet de route ont déjà paru dans mes articles sur Baudouin. Dans cet article, je cite les passages à partir du manuscrit (tapuscrit). Il s'ensuit que je n'indiquerai pas ici si le passage a déjà été publié dans un de mes articles.

4. Ibid., 11 octobre 1915, f. 4. 
longuement la scène qu'il lui fut donné à voir: «Et en même temps que cet olympien, que ce héros, qui allait être fixé dans la pierre, nous avions devant nous l'homme tout simplement, le même qui faisait son marché à Lucerne... » ${ }^{5}$. Avant de quitter Spitteler, Baudouin lui demande l'autorisation de traduire ses poèmes. L'autorisation lui est donnée, et Baudouin de conclure: «Le lien est noué.» ${ }^{6}$.

Le premier entretien de Baudouin avec Rolland a lieu le 25 octobre à l'hôtel Beau-Séjour, à Genève. Il évoque cette rencontre dans son Carnet de route. Nous y voyons dès le premier abord l'emprise de l'écrivain sur le jeune homme. Ce sont les yeux de Rolland qui frappent avant tout Baudouin:

Je crois bien n'avoir jamais vu des yeux si lumineux, si profondément limpides en même temps quallumés d'un éclair; c'est un bleu humide de lac sous un grand soleil; c'est toute l'intelligence et toute la passion; je ne sais quelle pureté fraîche et brûlante 7 .

Une spiritualité surhumaine paraît l'habiter. Baudouin évoque leur conversation dont le «ton» est celui «de la confidence, presque de la confession".

Il [Rolland] dit la tragédie de la guerre et sa propre souffrance, les amitiés anciennes éprouvées à ce nouveau creuset, les déceptions, la vie nouvelle qu'il faut se refaire sur des ruines.

Se remémorant les paroles de l'écrivain, Baudouin le cite:

Quand on me jette l'épithète d'humanitaire, [...] la malveillance se double d'un malentendu. Je sais bien que l'humanité s'en tirera. Ce n'est pas pour elle que j'ai peur. Mais c'est pour nos pays, pour mon pays ${ }^{8}$.

Rolland fait confiance à ce jeune homme de vingt-deux ans qu'il rencontre pour la première fois, et ceci d'autant plus que Baudouin lui parle de Carl Spitteler et des traductions de son œuvre qu'il a entamées.

5. Ibid., f. 6.

6. Ibid., f. 11.

7. Ibid., 25 octobre 1915, f. 31.

8. Ibid. Les mots en italique sont soulignés dans le manuscrit. 
Alors - écrit Baudouin - son regard s'illumine d'un de ces beaux éclairs et rejoint le mien. Je sens qu'à ce moment précis, il cesse de se tenir sur la défensive?.

L'amitié entre Baudouin et Rolland se noue autour de l'admiration qu'ils portent tous les deux à Carl Spitteler.

Rolland s'était, pour sa part, rendu à Lucerne en août 1915 pour rencontrer Spitteler. Sa visite fut motivée par l'admiration qu'il portait non seulement à l'ouvre poétique de ce dernier, mais aussi à son courage sur le plan politique. Dans un discours retentissant "Notre point de vue suisse", prononcé à Zurich le 14 décembre 1914, Spitteler avait osé flétrir publiquement la politique allemande et sa violation de la neutralité belge. Rolland s'identifia alors à Spitteler. Selon lui, le sens profond de son œuvre poétique est «la Révolte de l'homme isolé, qui refuse d'échanger son Ame libre contre la Conscience morale, officielle [...] que lui offre [...] le Maître, - l'Etat ou Dieu " ${ }^{10}$. Dans cet essai de 1925, publié à l'origine dans la revue Europe, quelques mois après la mort de Spitteler, Rolland écrit:

[...] quand je rencontrai celui qui a sacrifié sa vie à son âme, le héros, Prométhée [...] [je] fus foudroyé de joie et d'émotion. Je n'étais plus seul. J'avais un maître et compagnon ${ }^{11}$.

Spitteler obtiendra en 1919 le Prix Nobel de littérature, et ceci avec le soutien de Rolland. La lettre que Rolland avait écrite le 23 février 1918 au secrétaire de la Fondation Nobel pour appuyer la candidature du poète révèle l'emprise que Spitteler exerçait sur lui. Citons-en un extrait:

La lecture des grands poèmes de Spitteler a été pour moi une découverte, pendant la guerre, et je puis dire qu'elle m’a illuminé ces sombres années. J'avais le sentiment d'avoir fait la rencontre d'une de ces puissances personnalités de l'art, comme on soupire de n'en trouver que quelques-unes seulement, très rares, ça et là, dans le passé ${ }^{12}$.

9. Ibid., f. 32.

Io. R. Rolland, «Souvenirs et entretiens de Carl Spitteler», p. 181.

II. Ibid., p. 183.

I2. R. Rolland, Journal des années de guerre 1914-1919, p. 1415 sq. 
Comme Rolland le dit dans son essai de 1925, s'il avait trente ans de moins, il n'hésiterait pas à passer quelques années à traduire l'œuvre de celui qu'il place dans la "lignée de Goethe et de Milton" ${ }^{13}$. "Mais", écrit-il, "je sais qu'un autre y travaille; et nul ne le fera avec un plus pieux amour ${ }^{14}$. Rolland se réfère ici à Baudouin. En 1915, Baudouin n'en était qu'à ses débuts, mais il passera sa vie à traduire l'œuvre de celui qu'il considérait également comme un de ses "pères" spirituels. Deux jours après la mort de Spitteler, le 29 décembre 1924, Baudouin écrit dans son Carnet de route: "l'un des grands poètes du monde vient d'entrer dans sa patrie éternelle» ${ }^{15}$.

\section{Le Carmel et Romain Rolland}

Baudouin revoit Rolland début janvier 1916 pour lui parler de la revue Le Carmel qu'il est sur le point de fonder et qui se veut une tribune où puissent s'exprimer les voix libres d'Europe ${ }^{16}$. Il aimerait s'assurer sa collaboration active. Rolland refuse cependant de collaborer ouvertement à la revue. Il craint toute association officielle qui limiterait sa liberté d'esprit. Mais il sera d'une grande aide à Baudouin : non seulement il lui servira de conseiller, mais il le mettra en contact avec le réseau pacifiste qui s'est formé autour de lui en Suisse et avec des écrivains indépendants de langue allemande. Baudouin prendra le Prométhée de Spitteler comme symbole de sa revue. Il représente pour lui comme pour Rolland "le solitaire qui s'érige en face du troupeau» ${ }^{17}$.

Même si Rolland refuse de figurer parmi les " "patrons" " littéraires du Carmel $^{18}$, il ne refuse pas d'y être publié. C'est ainsi que Baudouin y fait paraître en décembre 1916 son article «La Route en lacets qui monte»

I3. R. Rolland, "Souvenirs et entretiens de Carl Spitteler», p. 181.

I4. Ibid., p. 182.

I5. Ch. Baudouin, Carnet de route, "Au-devant du monde", 31 décembre 1924, f. 139.

I6. Le premier numéro sort en avril 1916. La revue cesse de paraître en juin 1918. Pour une analyse détaillée du Carmel, voir les deux articles de A. Blum, «L'Europe, vue du Carmel (1916-1918)» et "Le Carmel, 1916-1918».

17. Ch. Baudouin, Carl Spitteler, p. 40.

I8. R. Rolland, lettre du 10 février 1916 à Baudouin, in A. Blum (éd.), Correspondance entre Romain Rolland et Charles Baudouin, p. 37. 
et publie également en avril 1918, le long essai de Rolland, Empédocle d'Agrigente et l'âge de la haine dans un des Cahiers du Carmel ${ }^{19}$. Baudouin contribue ainsi à faire connaître la pensée de Rolland pendant une période où certains périodiques suisses - tel le Journal de Genève ${ }^{20}$ entendent prendre leur distance à l'égard de cet écrivain encombrant.

Rolland tente - dans ses textes - de montrer comment la haine qui ravage le monde doit nécessairement céder la place à une forme d'Unité. "Tout travaille à notre idéal», écrit-il dans "La Route en lacets qui monte", "même ceux dont les coups s'efforcent à le ruiner» ${ }^{21}$. Il dessine ici l'avenir de l'Europe qui sera une véritable patrie formée par l'union des nations de l'Occident. Mais la patrie - l'Europe - ne représente pour Rolland qu'une première étape de «la fusion future des deux moitiés du monde, de deux hémisphères de la pensée: l'Europe et l'Asie»"22. Dans son essai sur Empédocle, dédié à Spitteler - "qui, par delà vingt siècles, renoua la tradition des poètes-philosophes d'Ionie» - Rolland veut donner un fondement philosophique à sa croyance que le monde et l'histoire sont soumis à des convulsions cosmiques, que "l'empire de la Haine" cédera la place à "celui de l'Amour» ${ }^{23}$.

\section{Hommages de Baudouin à Rolland pendant la Grande Guerre}

Rendant hommage à Rolland, Baudouin rédige à son tour en 1918 un essai intitulé "L'Empédocle de Romain Rolland». Il prend comme point de départ l'essai de Rolland pour nous montrer qu'il est également cet homme universel que ce dernier voit en Empédocle. Il est bien plus que l'auteur d'«Au-dessus de la mêlée»:

19. Chaque brochure des Cahiers du Carmel reprend soit un texte estimé important déjà publié dans les numéros précédents de la revue, soit une œuvre inédite.

20. Dix des seize articles que Rolland publia entre août 1914 et août 1915 parurent dans le Journal de Genève, mais dès la fin de 1915, il n'était plus le bienvenu au Journal. Seuls deux articles sur vingt-quatre parus entre décembre 1915 et juin 1919 seront publiés par ce périodique. Voir A. Blum, "Romain Rolland au cour de la "mêlée" ", p. 199-203.

2I. R. Rolland, «La route en lacets qui monte», p. 194.

22. Ibid., p. 191.

23. R. Rolland, «Empédocle d'Agrigente», p. 38. 
Il faut - insiste Baudouin - comprendre la signification de Romain Rolland [...] Comprendre à quel point Romain Rolland est un homme qui comprend, combien cette sensibilité est une intelligence assérénée [sic] ou lumière hellénique, arcboutée de vigueur moderne - combien il est aussi une "arche féerique» entre mondes ennemis, entre mondes épars - combien cet Occidental comprend lui aussi l'Orient où il voudrait nous voir regarder un peu plus ${ }^{24}$.

Son maître est un "philosophe» et "un encyclopédiste» 25 .

Dans le Carnet de Route de la même année, le portrait que Baudouin dresse de l'écrivain est également idéalisé malgré son vieillissement, vieillissement qu'il avait déjà remarqué l'année précédente. Le Rolland qu'il retrouve à Villeneuve, à l'Hôtel Byron, en juillet 1918, lieu de séjour de Victor Hugo et de Wagner, et où l'écrivain s'est installé en mai 1917, n'est plus - écrit Baudouin - «ce jeune chevalier clair, dont j'eus le privilège d'avoir une vision fugitive [en 1915]. Rolland n'a plus rien d'Olivier ${ }^{26}$. Ses traits son burinés par la "souffrance» ${ }^{27}$. Souffrance physique ou souffrance morale due aux combats qu'il mène? Nous ne savons pas. Mais elle ne fait qu'accroître l'admiration que Baudouin lui porte: "Jamais», affirme-t-il, «je n'eus à ce degré le sentiment d'avoir devant moi un produit d'élite de la civilisation humaine " ${ }^{28}$. L'écrivain est également un penseur politique pour Baudouin:

Quand Romain Rolland parle du présent politique, et essaie de prévoir l'avenir, il le fait comme je ne l'ai entendu faire à personne. Il est sur un autre plan; on dirait qu'il découvre toute la perspective. [...] C'est le libre esprit ${ }^{29}$.

Les hommages que Baudouin rend à Rolland peuvent aussi prendre une forme poétique. Son premier poème dédié à Rolland date du 21 novembre 1916. Il fut écrit pour féliciter l'écrivain qui venait de recevoir le Prix Nobel de littérature pour 1915, considéré par beaucoup comme un Prix Nobel de la paix. Baudouin décrit le parcours politique de Rolland en termes religieux. Soutenu par sa foi en sa mission, une foi

24. Ch. Baudouin, «L'Empédocle de Romain Rolland», f. 2 sq.

25. Ibid., f. 3.

26. Ch. Baudouin, Carnet de route, «La maison sur la route», 26 juillet 1918, f. 144.

27. Ibid., f. 145.

28. Ibid.

29. Ibid., f. 146 sq. 
qui pourrait s'apparenter à une foi «religieuse», Rolland - le pèlerin solitaire - parvient à vaincre les doutes de celles et ceux qu'il rencontre sur son chemin et à rassembler autour de lui des disciples:

$[\ldots]$

Vous étiez un pèlerin blanc parmi deux ou trois autres dans l'atmos-

$[\ldots]$ phère diaphane

Mais de choisir la foi quand le doute s'impose, c'était là votre courage.

C'était là votre courage, de prendre le chemin de l'exil volontaire,

[...]

Et de près ou de loin nous vous avons suivi par l'étrange route,

Tantôt dans la foi flamboyante et tantôt dans les affres du doute.

[...]

Et là, des centaines de mains belles vous attendent et vous tendent des

$[\ldots]$

palmes

Et pour vous accueillir de leurs hymnes, ces centaines de lèvres s'entr'ouvent.

Mon hommage parmi tous les autres ne serait rien s'il n'était qu'un hommage,

Mais il veut être un souvenir de la rencontre au temps plus solitaire du $[\ldots]^{30}$. pèlerinage,

\section{Baudouin, défenseur de son maître}

Baudouin - l'essayiste et le poète - peut aussi transformer sa plume en un instrument de combat et devenir un polémiste. Le disciple militant de Rolland prend la défense de son maître, objet d'attaques de celles et de ceux qui donnent dans la propagande française. Il publiera en août 1918 dans un des Cahiers du Carmel une brochure, Romain Rolland calomnié. Réponse à une diffamation et analyse d'une méthode simple de suggestion collective en réponse à un pamphlet publié à Genève en mai 1918,

30. Ce long poème, "A Romain Rolland" a été publié dans son intégralité dans l'Appendice (Document 1) d'A. Blum (éd.), Correspondance entre Romain Rolland et Charles Baudouin. La première partie du poème a paru dans le recueil poétique de Baudouin, L'arche flottante, p. 30 sq. 
intitulé Monsieur Romain Rolland, initiateur du défaitisme d'Isabelle Debran, journaliste suisse qui donnait dans la propagande française. La longue préface est d'un certain Diodore, pseudonyme sous lequel se cachait, selon Rolland, soit Paul-Hyacinthe Loyson ${ }^{31}$ qui menait en France, depuis le début de la guerre, une campagne contre Rolland, soit le nationaliste suisse, Robert de Traz, imprégné de Barrès et de Maurras. Une grande publicité avait été faite autour de cette brochure dans la presse suisse - dans le Journal de Genève et dans la Freie Zeitung.

C'est Rolland qui avait remis entre les mains de Baudouin les documents et montré du doigt les faux contenus dans le pamphlet, et Baudouin de s'exclamer dans son Carnet de route: «... c'est encore plus odieux et perfide que je ne pensais. " Il se fait donc un devoir d'y répondre, et dit: «Je n’ai jamais jamais aimé le pamphlet, je me le suis toujours défendu. Mais cette fois, il fallait, une incoercible indignation me poussait. " ${ }^{32}$. Diodore accuse Rolland d'abuser de son droit d'asile. Debran, quant à elle, reproche à Rolland ses mauvaises fréquentations : il entretient des liens étroits avec le Bund Neues Vaterland, premier mouvement allemand contre la guerre, avec Henri Guilbeaux et sa revue Demain $^{33}$, "revue défaitiste-bolchéviste " ${ }^{34}$, et avec Les Tablettes ${ }^{35}$, périodique "anarchiste» rédigé par des "déserteurs français» ${ }^{36}$. Dès la première phrase, Baudouin part à l'attaque:

Je ne suis point pamphlétaire. Je hais la polémique. Mais cette fois, c'en est trop. Non qu'il s'agisse de moi: $\mathrm{Ni}$ de près, ni de loin, je ne suis pris à partie. Et c'est justement cela qui m'engage à parler.

Il s'agit de plus que moi. Il s'agit même plus que de la défense d'un homme - d'un homme qui se tait et ne se défend pas. La machine calomniatrice que je vais démonter est un merveilleux spécimen. Il est

3I. Sur Loyson et les attaques violentes dont Rolland est l'objet, voir R. Roudil, "Romain Rolland et la "Bande à Loyson" ".

32. Ch. Baudouin, Carnet de route, «La maison sur la route», f. 148.

33. Henri Guilbeaux (1884-1938), pacifiste et internationaliste français est l'un des premiers défenseurs de Rolland pendant la Guerre. Sa revue Demain devint peu à peu l'organe du zimmerwaldisme de gauche, courant pacifiste révolutionnaire au sein du parti socialiste.

34. I. Debran, Monsieur Romain Rolland, initiateur du défaitisme, p. 28.

35. Revue fondée en 1916 par l'anarchiste et tolstoïen français, Claude Le Maguet, et le graveur et dessinateur belge, Frans Masereel.

36. I. Debran, Monsieur Romain Rolland, initiateur du défaitisme, p. 30. 
difficile de faire tenir, en si peu d'espace, plus de vérités maquillées, déguisées, d'un déguisement enfantin et qui fait pitié. C'est la calomnie-type, la calomnie adaptée à l'esprit du public moderne, ce public mitraillé de journaux et d'écrits où il n'a pas le temps de discerner, si bien que son discernement, à force de ne plus s'exercer, se rouille, et, chez beaucoup, ne peut plus fonctionner ${ }^{37}$.

Plus loin dans sa brochure, Baudouin évoque non seulement les accusations à l'encontre de Rolland, mais aussi la tactique des insinuations: «Insinuer: s'infiltrer en sinuant; le geste du serpent. Ce geste convient bien à certains polémistes. Cette peau leur va comme un gant. ${ }^{38}$.

Baudouin ne défend pas ici uniquement Rolland, mais tous ceux qui sont victimes de calomnies déguisées qui infectent les organes destinés au grand public peu averti. Par sa brochure il s'est "attiré des inimitiés» ${ }^{39}$, dont celle du sculpteur James Vibert avec qui il s'était lié d'amitié, et qui maintenant rompt avec lui.

Rolland ne put qu'être reconnaissant à Baudouin d'avoir pris sa défense. Il lui écrit à ce sujet le 11 août 1918 :

Votre Réponse n'est pas seulement une rude raclée, que vous administrez au trio ${ }^{40}$ de la Calomnie; c'est un maître morceau de littérature, solidement, sobrement, nerveusement écrit. Je l'ai lu deux fois - une première, par plaisir d'écrivain, - et la seconde, par plaisir personnel. Je vous remercie de tout cœur ${ }^{41}$.

Spitteler, quant à lui, attendra un an avant d'exprimer, également, son admiration pour sa «magnifique défense de Romain Rolland qui [1]'a enchanté par son style mâle et courageux». Dans une lettre à Baudouin datée du 15 juillet 1919, il explique pourquoi il ne put à l'époque s'associer à sa défense de Rolland:

Si j’ai gardé le silence, c'est que je déplore les idées politiques de notre cher ami, et plus encore ses accointances fâcheuses, les Guilbeaux, les

37. Ch. Baudouin, Romain Rolland calomnié, p. 5.

38. Ibid., p. 15.

39. Ch. Baudouin, Carnet de route, «La maison sur la route», f. 154.

40. I. Debran, Diodore et sans doute P.-H. Loyson.

4I. R. Rolland, lettre du 11 août 1918 à Baudouin, in A. Blum (éd.), Correspondance entre Romain Rolland et Charles Baudouin, p. 83. 
Debrits ${ }^{42}$, etc. [...] Démêler mon affection et mon admiration pour l'homme et le maître avec mes réserves concernant le pacifiste bolchévisant ce serait trop long et trop difficile pour moi ${ }^{43}$.

\section{Liber Amicorum Romain Rolland 1926}

A l'automne de 1925, Baudouin est appelé à participer au Liber Amicorum qui sera offert à Rolland pour ses soixante ans. Une petite fête est prévue à l'Hôtel Byron pour le 31 janvier 1926. C'est à travers un poème, un "Triptyque» dans lequel il s'adresse directement à l'écrivain, que Baudouin entend exprimer son "affection" et sa "gratitude" pour le soutien affectif, intellectuel et littéraire que Rolland lui a offert généreusement depuis dix ans.

Dans son poème Baudouin évoque l'après-midi de juillet 1918 passé à Villeneuve, le souvenir de cette rencontre étant encore «si vif - frais et brûlant " ${ }^{4}$. Il «revoi[t] un jour d'été / Au-dessus du lac qui vibre / parmi les feuilles du verger " ${ }^{45}$ et les "éclairs " ${ }^{46} \mathrm{du}$ lac qui se reflètent dans les yeux de Rolland. Comme lors de sa première rencontre avec Rolland, le regard, les yeux de l'écrivain le frappent. C'est «ce dur regard lucide / du libre esprit au vol d'aigle», "cet incisif regard / cruel à force de clarté» ${ }^{47}$, c'est-à-dire - le regard de celui qui a vu clair pendant toute la durée de la Guerre. Le ton du poème est religieux comme l'était celui de 1916, déjà cité: "Mil neuf cent dix-huit: c'est l'heure / la plus pantelante du meurtre, / le De Profundis d'Europe. " ${ }^{48}$. Rolland apparait à nouveau ici comme une figure christique. Baudouin évoque chacun de ses gestes. Ses mains le font songer à "d'autres mains / posées sur des fronts d'enfants", et sur son visage. "J'ai vu», conclut-il, «vous aviez des larmes / lorsque

42. Jean et Marguerite Debrit. Ce dernier était directeur de l'hebdomadaire socialiste genevois, La Nation.

43. C. Spitteler, lettre du 15 juillet 1919 à Baudouin, citée dans A. Blum (éd.), Correspondance entre Romain Rolland et Charles Baudouin, n. 4, p. 133.

44. Ch. Baudouin, Carnet de route, "Au-devant du monde», 31 octobre 1925, f. 161.

45. Ch. Baudouin, "Tryptique», p. 40.

46. Ibid., p. 41.

47. Ibid., p. 42.

48. Ibid., p. 40. 
vous parliez du monde et de sa grand pitié» ${ }^{49}$. Rolland représente pour Baudouin celui qui a sacrifié sa vie et sa personne pour l'humanité.

\section{Baudouin à Villeneuve}

Baudouin se rend à nouveau à Villeneuve le 3 avril 1929, cette fois-ci à la Villa Olga où Rolland s'est installé en 1922. Il y décrit une atmosphère de recueillement propre à ce saint laïc qu'est encore l'écrivain à cette époque pour lui. «Le silence est son climat», silence que même les chats respectent:

[...] toute une tribu de chats bien élevés vont et viennent à pas feutrés, passage de fourrures douces, lutins tranquilles, comme respectueux du maître, autour duquel il semble naturel que tout fasse silence ${ }^{50}$.

Baudouin note la "voix matériellement faible» de Rolland, "mais qui enregistre tous les mouvements de l'énergie", voix "qui n'est bien à son aise que dans l'intimité du tête-à-tête, et qui exige le silence pour être entendue " ${ }^{51}$. Et c'est cette intimité que Rolland accorde à son jeune ami depuis leur première rencontre. Baudouin sait par des tiers que Rolland l'aime et l'estime, d'où les moments privilégiés qu'il partage avec lui. Et pourtant, avouera le disciple en 1931 :

[...] Romain Rolland m'intimide toujours. Son extrême bonté n'exclut pas la distance, qu'il garde toujours jusque dans l'intimité même, par un instinct de pudeur, de noblesse, de fierté indéniable, et qui d'ailleurs ne blesse jamais, car elle vient de haut ${ }^{52}$.

Toute étude sur Baudouin et Rolland serait incomplète si l'on n'évoquait pas la mémorable journée que Baudouin passa à Villeneuve en décembre 1931, invité par Rolland pour y rencontrer Gandhi, journée dont Baudouin rend compte en détail dans son Carnet de route. Il est intéressant d'y lire la comparaison qu'il dresse entre Rolland et

49. Ibid., p. 42.

50. Ch. Baudouin, Carnet de route, "Paris-quatre-Saisons», 3 avril 1929, f. 19 (pagination multiple).

5I. Ibid.

52. Ch. Baudouin, Carnet de route, "Passage du méridien", 12 décembre 1931, f. 35. 
Gandhi - comparaison qui peut être considérée comme une autre forme d'hommage à Rolland.

Baudouin pénètre pour la première fois dans la chambre de Rolland où seront prises les photos désormais emblématiques du tête-à-tête entre Rolland et Gandhi. Il est le seul des amis de Rolland à être présent. Baudouin est conscient de son privilège : "Il est beau», écrit-il, "de voir cette interrogation mutuelle des regards de ces deux grands hommes, en qui s'incarnent le meilleur de l'Occident et de l'Orient. ${ }^{53}$. Vêtu de "sa grande cape grise», Rolland a "un air princier». Baudouin scrute «les deux profils» qui «s'affrontent. Celui de Rolland, très clair et remarquablement élevé, fait paraître, par contraste, plus brun et plus ramassé celui de Gandhi...» ${ }^{54}$. Baudouin note l'ouverture d'esprit de Rolland: «[...] toute l'interrogation [...] un peu anxieuse" "paraît" de son "côté»; "Gandhi est beaucoup plus fermé sur lui-même; [...]". "Certes il écoute; certes, il observe; mais c'est nonobstant un homme de doctrine et d'action; son siège est fait; [...] ». Par contre, "Rolland, en face de lui, un peu incliné, est tout accueil; c'est l'âme disponible, habile dans l'art de la sympathie, prête à épouser la forme de l'autre» ${ }^{55}$.

\section{La remise en question du Maître}

Mais c'est justement à partir de 1931 que le Disciple prend ses distances à l'égard du Maître. Il ne peut le suivre dans son évolution politique. Il est troublé par une lettre que Rolland lui a écrite le 31 janvier 1931, dans laquelle il prend la défense de l'URSS "contre un "Européanisme" très suspect", soutenu par le capitalisme occidental et les intellectuels «idéalistes ${ }^{56}$. Baudouin tentera, néanmoins, au long de ces années 30 de comprendre celui qu'il "prenai $[t]$ " jusqu'à cette époque "pour un Dieu» et qu'il «tenai $[t]$ pour infaillible " ${ }^{57}$. Comprendre Rolland, c'est une façon pour Baudouin de ne pas s'éloigner de son maître.

53. Ibid., f. 32.

54. Ibid., f. 31.

55. Ibid., f. 32.

56. R. Rolland, lettre du 31 janvier 1931 à Baudouin, in A. Blum (éd.), Correspondance entre Romain Rolland et Charles Baudouin, p. 178.

57. Ch. Baudouin, lettre du 12 mars 1931 à Rolland, in A. Blum (éd.), Correspondance entre Romain Rolland et Charles Baudouin, p. 181. 
Baudouin continue à rendre visite à Rolland à Villeneuve. Il le revoit en décembre 1935, après son retour de l'Union soviétique. Son maître est sans aucun doute toujours pour lui, comme il l'était en 1931, un "grand seigneur de l'esprit " ${ }^{58}$. Il le revoit également en 1936, mais note que maintenant «le vieillissement» de Rolland influe sur son caractère. Une forme d'" exacerbation» se manifeste chez lui:

Je reconnais là - dit Baudouin - le "pli névralgique» que j'ai souvent vu apparaître sur son visage, [...] comme la réaction ombrageuse à un mot, à une idée, et qui maintenant s’accuse, se répète, s’approfondit. Il ne faut pas discuter, pas insister...

Mais le Rolland qu'il a devant lui ne doit pas lui effacer le Rolland qu'il a tant admiré:

[...] il ne faut surtout pas permettre que ces petites choses diminuent la grande image que nous avons de lui ${ }^{59}$.

Baudouin parle pour lui-même et pour les autres.

Et pourtant, la position politique de Rolland n'est plus «tout à fait à la hauteur du Rolland de la grande époque - qui reste celle de la guerre». Rolland en est-il conscient? Baudouin croit déceler chez lui une forme de «mauvaise conscience»: «Il ne paraît pas tout à fait d'accord avec luimême", d'où son irritation à l'égard de ceux qui tentent de s'opposer à lui. Mais, comme pour excuser son maitre, Baudouin ajoute:

Mais qui peut se vanter, dans le chaos présent, d'être d'accord avec soi, quand les choses elles-mêmes ne sont pas d'accord entre elles? ${ }^{60}$

\section{Liber Amicorum 1936}

Malgré leur profonde divergence politique, à aucun moment il n'y a eu rupture entre les deux hommes, comme ce fut le cas, par exemple, entre Rolland et Panaït Istrati ou Marcel Martinet - ceci grâce avant tout à

58. Ch. Baudouin, Carnet de route, "Passage du méridien», f. 31. C’est ainsi que Baudouin avait caractérisé Rolland en 1931.

59. Ch. Baudouin, Carnet de route, "A la croisée des branches", 7 août 1936, f. 145.

6o. Ibid. 
Baudouin qui n'ose pas ou se refuse à affronter son Maître, ou, s'il le fait, c'est sur la pointe des pieds. Le dévouement de Baudouin est tel qu'il va même jusqu'à se porter volontaire pour la mise sur pied et la sortie d'un Hommage collectif pour les soixante-dix ans de Rolland. Le volume ne verra pas le jour, non seulement pour des raisons matérielles, mais peutêtre aussi, avant tout, à cause des tensions qui s'étaient installées entre Rolland et les pacifistes pour qui il avait été un maître à penser. Une lecture des lettres échangées entre Rolland et Baudouin à ce sujet nous montre un Rolland difficile: il entend contrôler le contenu de l'Hommage. Si l'Hommage ne correspond pas à ses exigences, il demande à ce que Baudouin y renonce. Dans sa lettre du 27 janvier 1936, Rolland écrit à Baudouin:

[...] je ne juge pas que les témoignages français que vous avez recueillis suffisent [...] Vous vivez en dehors de l'heure présente; vous êtes resté au temps de notre petit groupe de pacifiste de 1917, à Genève [...]. Si vous m'aviez consulté, je vous aurais prié de laisser certains noms en dehors de votre consultation ${ }^{61}$.

Rolland ne peut accepter des hommages venant de personnes critiques à l'égard de son compagnonnage de route avec l'Union soviétique. Quelques jours plus tard, il insiste:

[...] il est absolument indispensable d'élargir beaucoup les dimensions, un Liber Amicorum 1936 R.R. serait inconcevable si ne s'y exprimaient en première place ceux qui marchent d'accord avec le R.R. $1936^{62}$.

Même si un Liber Amicorum ne paraît pas en 1936, il est intéressant de lire la préface que Baudouin avait préparée pour le recueil. Il évoque - le 20 janvier 1936 - tous ceux qui ont répondu avec une «spontanéité émouvante" ${ }^{63}$ à son appel de textes. Il a été ému "de voir, en quelques jours, affluer vers le relais de $[s]$ a petite maison ${ }^{64}[\ldots]$ nombre de pages vivantes et palpitantes, qui se pressaient comme si elles n'attendaient que

6I. R. Rolland, lettre du 27 janvier 1936 à Baudouin, in A. Blum (éd.), Correspondance entre Romain Rolland et Charles Baudouin, p. 211 sq.

62. R. Rolland, lettre du 2 février 1936 à Baudouin, in A. Blum (éd.), Correspondance entre Romain Rolland et Charles Baudouin, p. 217.

63. Ch. Baudouin, " $70^{\mathrm{e}}$ anniversaire de Romain Rolland", f. 5.

64. La maison de Baudouin à Saconnex d'Arve, près de Genève. 
cet appel " ${ }^{65}$. S’adressant directement à Rolland dans sa conclusion, le disciple écrit :

Que votre seul nom soit prononcé, et c'est le signe de ralliement de tous ceux qui savent être présents, là où il sied de l'être. Tous ne partagent pas vos idées, votre foi. Mais tous ont entendu votre appel au héros, votre appel à l'humain; [...] vous êtes celui par qui l'héroïque devient humain et l'humain héroïque ${ }^{66}$.

Baudouin suggère toute en douceur le fait que l'évolution politique de Rolland ne fait pas l'unanimité, mais déclare qu'au-delà de ces différences reste une dette de reconnaissance que tous ont envers lui.

Quoique ce Liber Amicorum reste lettre morte, Baudouin prendra en charge l'organisation d'une soirée à Genève, au Victoria Hall, en l'honneur des soixante-dix ans de Rolland. Elle se déroulera le 23 avril 1936 sous l'égide de l'Association des Intellectuels pour la Défense de la Culture. Cette soirée paraît avoir été de nature purement littéraire et musicale si l'on en juge par le programme. Tel ne fut pas le cas dans la France du Front Populaire où, au contraire, l'anniversaire de Rolland fut fêté en grande pompe le 31 janvier à Paris à la Mutualité et transformé en manifestation politique. A l'occasion de cette soirée genevoise, Baudouin compose un poème intitulé "Christophe» où il dresse l'image de Saint-Christophe:

$[\ldots]$

Christophe de son pas a traversé longtemps.

La rive d'autrefois est loin derrière lui,

Et son pied déjà touche aux bords de l'avenir.

$[\ldots]^{67}$

En effet, Rolland a abandonné la position qu'il défendait en 1919 - celle énoncée dans sa "Déclaration d'indépendance de l'esprit» pour se lancer sur une autre rive - celle dessinée par l'Union soviétique - la nouvelle société de l'avenir.

65. Ibid., f. 1.

66. Ibid., f. 5.

67. Ch. Baudouin, "Christophe», publié en entier dans l'Appendice (document 3) d'A. Blum (éd.), Correspondance entre Romain Rolland et Charles Baudouin. 
Trois jours plus tard - le 26 avril - Rolland adresse à Baudouin une lettre de remerciements et lui demande si le poète et dramaturge vaudois, René Morax est venu à la soirée en son honneur et il ajoute: «J'aimerais à savoir ce qu'il a dit [....] Car c'est la première fois que nous nous retrouvons de nouveau, après vingt ans d'interruption d'une vieille amitié.» ${ }^{68}$. Rolland pense sans doute ici à leur collaboration en 1914 pour la mise sur pied d'un numéro spécial des Cahiers Vaudois pour protester contre le bombardement par les Allemands de Louvain et de Reims. Morax n'est pas venu à la soirée, mais il a adressé un long message sous forme d'une très belle lettre ouverte à Rolland. Morax rappelle ce qu'il lui doit. Le Théâtre du peuple de Rolland, paru en 1903, l'aida à créer en 1908 son théâtre du Jorat. Morax y évoque la Suisse où Rolland est venu:

[s']établir au bord de notre lac, [...] où tant d'hommes illustres ont trouvé le repos ou la retraite, ou le renouvellement de leur énergie. Vous deviez connaître cet isolement de ceux que Spitteler appelait «les blaireaux solitaires». [...]

Pour dominer les passions qui aujourd'hui bouleversement le monde, [...] vous avez choisi cet observatoire ${ }^{69}$.

Et Morax continue: au long de ces années Rolland n'a "abandonné ni [sa] foi en l'avenir, ni [son] idéal de justice et de liberté... ». L'écrivain a "compris et réalisé» qu'il faut «gagner» et «défendre» la liberté "par la lutte et le sacrifice». Son appel a été entendu, d'où les nombreux témoignages qui affluent vers lui de tous les pays. Morax clôt sa lettre avec cette phrase: "Car la voix la plus écoutée n'est pas celle qui crie sur la place publique ou dans la radio, mais celle du solitaire. ${ }^{70}$.

En septembre 1937, Baudouin se rend à nouveau à Villeneuve pour y rencontrer Rolland. Une tristesse l'habite ainsi que la nostalgie de ce qui va disparaître. On détruit les ruines de l'Hôtel Byron, victime d'un incendie en 1933 - lieu de tant de souvenirs pour Rolland - celui de sa rencontre avec Victor Hugo à l'âge de seize ans et celui de la célébration de son anniversaire en 1926. Et maintenant Rolland se prépare à quitter Villeneuve pour regagner la Bourgogne. En souvenir de ce lieu de retraite, de silence, auquel il est très attaché, Rolland dédiera son

68. R. Rolland, lettre du 26 avril 1936 à Baudouin.

69. R. Morax, lettre du 23 avril 1936, f. 6.

7o. Ibid., f. 7. 
autobiographie spirituelle - Le Voyage intérieur - "A mon vieux noyer de Villeneuve, qui fut mon compagnon de songe ${ }^{71}$. Et Baudouin d'écrire ces phrases pleines d'affectivité:

Cet européen, cet esprit international entre tous, est fort attaché à son coin de terre; on sent qu'il ne voudrait pas mourir ailleurs. Je l'aime ainsi, il est plus complet, plus humain. Il faut que ses yeux se reposent à nouveau sur les parages que hanta Colas Breugnon ${ }^{72}$.

Rolland meurt le 30 décembre 1944 à Vézelay. Le lendemain Baudouin confie sa tristesse à son Carnet de route. Il réunit dans une même pensée ses deux grands maitres -l'Européen Rolland et le Suisse Spitteler:

Romain Rolland est mort, [...] vingt ans, presque jour pour jour, après Spitteler qu'il a tant aimé. C'est une grande tristesse. Il aura tenu bon durant ces cinq dernières années, et voilà qu'il tombe au dernier moment. Il n'aura pas vu la terre promise. Il me disait dans une de ses dernières lettres, son désir de causer ensemble, après toutes ces heures d'histoire que nous avons vécues... J'ai relu toutes ses lettres. C'est un trésor inestimable ${ }^{73}$.

\section{Hommage à Romain Rolland 1945}

Il reste maintenant à Baudouin de rendre un dernier hommage à Rolland en mettant sur pied un volume collectif à la mémoire de l'écrivain. Grâce à ses efforts, un Hommage à Rolland sortira en 1945 aux Editions du Mont-Blanc à Genève. Il y réunit des articles écrits en 1936, dont certains furent publiés à l'époque dans la presse, ainsi que de nouveaux textes - sous forme de messages et des poèmes. Comme Baudouin l'explique dans son avant-propos, il a voulu grouper dans son volume différents types de témoignages. A travers ces témoignages, ce sont les multiples visages de Rolland que Baudouin entend montrer. Pour certains, il fut avant tout l'auteur de Jean-Christophe, œuvre qui leur ouvrit "un des royaumes de la beauté» et, pour d'autres, à travers son article «Au-dessus

7I. R. Rolland, Le voyage intérieur (Songe d'une vie), p. 7.

72. Ch. Baudouin, Carnet de route, "Le charme noir de Perséphone», 2 septembre 1937, f. 82.

73. Ch. Baudouin, Carnet de route, «Intermezzo", 31 décembre 1944, f. 7. 
de la mêlée», il «soulag[ea] leur "conscience" ». Entre les deux guerres, il devint pour certains celui qui leur fit entrevoir les "promesses d'une société nouvelle » ${ }^{74}$, c'est-à-dire celles que semblait leur offrir l'Union soviétique, ou encore celui qui les guida vers la découverte de l'Inde mystique. Le dernier Rolland serait "celui de la Résistance " ${ }^{75}$. Ces témoignages sont précédés d'un texte de Baudouin: «Rencontres de Romain Rolland». Ce sont des extraits de son Carnet de route déjà évoqués dans cette étude.

Parmi les textes que Baudouin a reçus du monde entier, figurent des textes de Suisses, dont ceux d'Alexandre Mairet et de Charly Guyot. Certains témoignages ne furent pas retenus pour le volume, tel celui de Lydie Malan, professeur au conservatoire de Genève.

Le graveur et peintre genevois Mairet connaissait Baudouin de longue date, depuis peu après son arrivée à Genève. Il avait fait partie de noyau fondateur de sa revue Le Carmel se chargeant, entre autres, des articles traitant des questions d'art. La couverture de la revue et sa vignette sont l'œuvre de Mairet. Mairet connaissait Rolland dès cette époque et rappelle dans son article que l'écrivain «avait été un des premiers amis du Carmel»" ${ }^{76}$. Voulant rendre "hommage" au rôle qu'avait joué Rolland pendant la Grande Guerre, Mairet raconte qu'il lui rendit visite à Genève, à l'hôtel Beau-Séjour où l'écrivain habitait au début de la guerre. Comme l'écrit Mairet: "Son courage qui lui valait tant d'inimitiés méritait qu'il fût soutenu approuvé, encouragé, par un témoignage d'affection et d'admiration. " Mairet qui d'habitude réservait son admiration pour celui qui travaille de ses mains plutôt que pour l'intellectuel " "en habit du dimanche" " tel que lui apparut au premier abord Rolland dut constater qu'il s'était mépris sur son compte: «[...] certaines personnes plutôt chétives ont parfois plus de caractère, plus d'énergie et plus de force morale que bien des êtres solides et charpentés. " ${ }^{77}$. Alors que l'amitié entre Baudouin et Rolland s'était forgée autour de leur admiration commune pour Spitteler, celle entre Mairet et Rolland se

74. Ch. Baudouin, "Avant-Propos», p. 7.

75. Ceux qui saluaient en Rolland un homme de la Résistance se leurraient. L'écrivain avait pris ses distances face à elle. Voir à ce sujet la biographie de B. Duchatelet, Romain Rolland tel qu'en lui-même et ses deux articles, "Romain Rolland face à la Seconde Guerre mondiale» et «Le second journal des années de guerre de Romain Rolland».

76. A. Mairet, «Message», p. 118.

77. Ibid., p. 119. 
forgea autour de leur admiration pour Jean-François Millet, et Mairet de dire: "Il était beau de voir que l'homme "en habit du dimanche" comprenait et sentait, lui, le peintre du peuple.» Et en conclusion Mairet écrit:

Millet et Tolstoï étaient présents entre Romain Rolland et celui [c'està-dire lui-même] qui lui apportait le modeste tribut de son hommage. Par eux, nous communiions en cet esprit de fraternité qui est au-delà du temps et "au-dessus de la mêlée » ${ }^{78}$.

Même si le témoignage de Lydie Malan, resté inédit, ne figure pas dans le volume, il évoque en quelques lignes poétiques un moment privilégié passé avec Rolland:

Romain Rolland ... ce nom évoque pour moi évocation de poésie. Image de lumière et de force. Etre vivant, dans le souvenir et dans l'audelà, comme aujourd'hui et sur la terre. Beauté simple et complexe. Inspiration.

Il y a quelques années nous sommes allés lui rendre un hommage musical; ce fut comme un conte de fées; par une journée de soleil, après le voyage sur le lac, nous montions l'allée sans parler, pénétrés de respect. Et il y avait là aussi Tagore ${ }^{79}$ et quelques autres. Nous n'en avons pas parlé; ce n'était pas "pour le monde»; et cela est resté mon plus beau souvenir du "Motet de Genève» ${ }^{80}[\ldots]^{81}$.

Tout comme Baudouin, dans des passages de son Carnet de route cités dans cette étude, Lydie Malan évoque la magie de ses rencontres avec Rolland à Villeneuve, dans un lieu baigné de lumière, qu'elle soit spirituelle - celle projetée par Rolland, qu'elle soit physique - celle du soleil se reflétant sur le lac.

Si les hommages rendus par Mairet et Lydie Malan sont de nature affective, tel n'est pas le cas de celui du critique littéraire et professeur neuchâtelois Charly Guyot, futur recteur de l'Université de Neuchâtel. Son texte intitulé "Style d'une vie» représente une analyse à la fois littéraire et intellectuelle de l'œuvre et de la pensée de Rolland. Son essai qui s’adresse

78. Ibid., p. 120.

79. Tagore rendit visite à Rolland en juillet 1926.

8o. Référence au «Motet de Genève» que L. Malan créa en 1926 au Conservatoire.

8I. L. Malan, texte non daté [1945 ?], in Hommage à Romain Rolland. 
à des lecteurs de 1945 peut encore nous interpeller aujourd'hui. Guyot tente d'expliquer et de s'expliquer les raisons pour lesquelles Rolland qui connut lors de la Guerre de 14 "la plus grande célébrité» est «environné» depuis la veille de la Seconde guerre "d'un demi-silence" ${ }^{82}$. Le critique montre l'effort que Rolland fit sa vie durant pour concilier les exigences d'ordre artistique et les exigences d'ordre politique et admire sa capacité de se remettre en question sur le plan politique dans sa recherche de la vérité. Rolland aurait donc été le premier - déclare Guyot - à exiger «un hommage sincère» ${ }^{83}$. Il s'ensuit que tout hommage doit montrer non seulement «la force», mais aussi la «faiblesse» de l'écrivain ${ }^{84}$. Lui reprochant ses personnages désincarnés, Guyot de conclure:

L'artiste, l'écrivain, sont, chez lui, partiellement vaincus. Mais l'homme divisé longtemps contre lui-même, faisant un suprême effort pour se rassembler, est de ceux qui font honneur à l'Homme. Quinze ans de combat ${ }^{85}$ imposent respect et admiration. L'œuvre la plus vivante, la plus exemplaire qu'ait composée Romain Rolland, ce n'est peut-être pas Jean-Christophe et moins encore L'Ame enchantée. C'est sa vie ${ }^{86}$.

Comme nous avons déjà vu, Baudouin aime célébrer Rolland en lui offrant des poèmes. Il en fait de même en 1945. C'est ainsi que dans cet Hommage figure son "Deuxième triptyque à Romain Rolland». Certains vers de ce poème ont également une teneur religieuse. Quoique Rolland n'y soit pas nommé, sa présence est suggérée par des images qui renvoient à la Passion du Christ. Il est celui qui ne dort pas - qui reste à l'écoute de ce monde qui souffre - contrairement à ses "apôtres" qu'il dut souvent réveiller. Baudouin s'adresse aux mânes de Rolland et tente de rassurer son maître: ceux qui furent ses disciples de son vivant sont toujours "présents» à son appel qui perdure. Cet Hommage collectif en est le témoignage:

82. Ch. Guyot, «Style de vie», p. 56.

83. Ibid., p. 60.

84. Ibid., p. 61.

85. Recueil d'articles politiques de Rolland écrits entre 1919 et 1934 et publié en 1935. Quinze ans de combat est précédé d'une longue introduction ("Adieu au passé») où Rolland explique son évolution politique.

86. Ch. Guyot, "Style de vie», p. 61 sq. 
Après ces dix et vingt ans,

Voyez nos cœurs qui sont sûrs

Des fidélités futures,

[...]

A cause de la lumière

Qui vit dans votre regard ${ }^{87}$.

[...]

Vous nous avez enseigné

Qu'il n'est nul salut pour soi

Tant que l'on verra saigner

L'Homme qui est mis en croix

[...]

Et qu'il faut, $[\ldots]$

[...]

Debout demeurer présent

A la misère du monde ${ }^{88}$.

En 1945, Baudouin rend ainsi un dernier hommage à son Maître rencontré trente ans plus tôt. Ce fut grâce à la Suisse, devenue au fil des ans sa «seconde patrie» qu'il avait pu, pourrions-nous dire, "servir», Rolland. Sur le «sol» suisse, Baudouin eut l'occasion de rencontrer - écrit-il dans sa "Note liminaire» de 1943 au Carnet de route - ces " "bons Européens" " qui n'étaient pas «ses fils", mais:

[...] qui furent les uns et les autres, de manière passagère ou durable, attirés, retenus, par la vertu de ce petit pays, confluent des civilisations et des races, où l'Europe, dans ses pires convulsions destructrices, sut préserver sa propre image ${ }^{89}$.

Parmi toutes ses rencontres, celle de Romain Rolland fut sans doute la plus importante.

Antoinette BLUM

New York

87. Ch. Baudouin, «Deuxième triptyque à Romain Rolland», p. 130. Le premier triptyque, dont nous avons cité quelques vers, est celui publié dans le Liber Amicorum de 1926 pour Rolland.

88. Ibid., p. 132.

89. Ch. Baudouin, «Note liminaire», février 1943, f. 2. 


\section{BIBLIOGRAPHIE}

\section{Fonds conservés à la Bibliothèque de Genève, salle des manuscrits}

Baudouin, Charles, Carnet de route, "Au-dessus de la mêlée», octobre 1915-septembre 1916, ms. fr. 5963, dossier 3, IV. Tapuscrit.

—, Carnet de route, "La maison sur la route», septembre 1916-octobre 1918, ms. fr. 5964, dossier 1, V. Tapuscrit.

-, Carnet de route, "Au-devant du monde», janvier 1922-avril 1926, ms. fr. 5964, dossier 3, VII. Tapuscrit.

_, Carnet de route, "Paris-quatre-Saisons", 1922-1937, ms. fr. 5965, dossier 8, X. Tapuscrit.

—, Carnet de route, "Passage du méridien», 1931-1933, ms. fr. 5966, dossier 2, XIV. Tapuscrit.

—, "Textes autobiographiques: "Connais-toi toi-même”", novembre 1932, ms. fr. 5959, env. 2.

—, Carnet de route, "A la croisée des branches», 1933-1936, ms. fr. 5966, dossier 3, XV. Tapuscrit.

—, Carnet de route, "Le charme noir de Perséphone», 1936-1939, ms. fr. 5966, dossier 4, XVI. Tapuscrit.

—, «70 anniversaire de Romain Rolland. Liber Amicorum», Adresse à Romain Rolland, à l'occasion de son $70^{\mathrm{e}}$ anniversaire, ms. fr. 6004, env. 15, document 3b, 20 janvier 1936.

—, «Note liminaire», février 1943, ms. fr. 5970, env. 2. Tapuscrit.

—, Carnet de route, "Intermezzo", 1944-1945, ms. fr. 5967, dossier 3, XIX. Tapuscrit.

—, "L'Empédocle de Romain Rolland», textes divers concernant des personnages, ms. fr. 6017/6, document 3 [sans doute 1918].

Malan, Lydie, in «Hommage à Romain Rolland», ms. fr. 6004, env. 20, document 18 [texte non daté].

Morax, René, lettre du 23 avril 1936 à Romain Rolland, ms. fr. 6005, env. 1, f. 1-7. 
Rolland, Romain, lettre du 26 avril 1936 à Charles Baudouin, Tapuscrit, Fonds Romain Rolland, NAF 27360, Département des manuscrits, Bibliothèque nationale de France, Paris.

\section{Sources éditées}

Baudouin, Charles, L'arche flottante, Cahiers du Carmel, $1^{\text {re }}$ série, $\mathrm{n}^{\circ} 2$, Paris, La Maison française d'art et d'essai, 1919.

-, "Avant-Propos", in Hommage à Romain Rolland, Genève/ Annemasse, Editions du Mont-Blanc, 1945, p. 7-9.

-, Carl Spitteler, essai suivi d'un choix de fragments en traduction originale, Bruxelles, «Les cahiers du journal des poètes», 53 (5 mai), 1938.

—, "Deuxième triptyque à Romain Rolland", in Hommage à Romain Rolland, Genève/Annemasse, Editions du Mont-Blanc, 1945, p. 129-132.

-, Romain Rolland calomnié. Réponse à une diffamation et analyse d'une méthode simple de suggestion collective, Cahiers du Carmel, $1^{\mathrm{er}}$ cahier, II ${ }^{\mathrm{e}}$ semestre, Genève/Paris, Imprimerie Reggiani/ Maison française d'art et d'édition, 1918.

—, "Triptyque», in Liber Amicorum Romain Rolland, témoignages recueillis par M. Gorki, G. Duhamel, S. Zweig, Zurich, Rotapfel Verlag, 1926, p. 40-42.

Blum, Antoinette (éd.), Correspondance entre Romain Rolland et Charles Baudouin: une si fidèle amitié: choix de lettres (1916-1944), avantpropos d'Yves Baudouin, Meyzieu, Césura, 2000.

Hommage à Romain Rolland, Genève/Annemasse, Editions du MontBlanc, 1945.

Rolland, Romain, «Empédocle d'Agrigente», in Compagnons de route, nouv. éd., Paris, Albin Michel, 1953, p. 23-58.

—, Journal des années de guerre 1914-1919, Paris, Albin Michel, 1952.

—, Quinze ans de combat, 1919-1934, Paris, Rieder, 1935.

—, "La route en lacets qui monte", in L'esprit libre: Au-dessus de la mêlée; les précurseurs, Paris, Albin Michel, 1953, p. 188-194.

—, "Souvenirs et entretiens de Carl Spitteler», in Compagnons de route, nouv. éd., Paris, Albin Michel, 1961, p. 179-198. 
—, Le voyage intérieur (Songe d'une vie), nouv. éd. augmentée de textes inédits, Paris, Albin Michel, 1959.

\section{Travaux}

Blum, Antoinette, Charles Baudouin, Romain Rolland. Une amitié à toute épreuve, Brèves, Association Romain Rolland, 2004 (Etudes Rollandiennes 3).

—, "Le Carmel, 1916-1918. Une revue genevoise d'inspiration européenne», La Revue des revues, 25 (1998), p. 67-81.

—, "L'Europe, vue du Carmel (1916-1918)», Equinoxe, 17 (printemps 1997), p. 37-53.

—, "Regard sur la Correspondance (1916-1944) entre Romain Rolland et Charles Baudouin", Action et pensée, 41 (décembre 2002), p. 37-52.

—, "Romain Rolland au cour de la "mêlée" ", in Genève et la paix. Acteurs et enjeux. Trois siècles d'histoire, éd. par Roger Durand, en collaboration avec Jean-Daniel Candaux et Antoine Fleury, Genève, Association "Genève: un lieu pour la paix", 2005, p. 193-211.

Debran, Isabelle, Monsieur Romain Rolland, initiateur du défaitisme, introduction par Diodore, Genève, Imprimerie Henry Jarrys, 1918.

Duchatelet, Bernard, Romain Rolland tel qu'en lui-même, Paris, Albin Michel, 2002.

—, "Romain Rolland face à la Seconde Guerre mondiale: l'année 1940 (d'après son Journal inédit)", in Romain Rolland, une auvre de paix, éd. par Bernard Duchatelet, Paris, Publications de la Sorbonne, 2010, p. 181-194.

—, "Le second journal des années de guerre de Romain Rolland", in Les journaux de la vie littéraire, Rennes, Presses universitaires de Rennes, 2009, p. 395-410.

Guyot, Charly, "Style de vie», in Hommage à Romain Rolland, Genève/ Annemasse, Editions du Mont-Blanc, 1945, p. 56-62.

Mairet, Alexandre, "Message», in Hommage à Romain Rolland, Genève/Annemasse, Editions du Mont-Blanc, 1945, p. 117-120. 
Roudil, Roland, "Romain Rolland et la "Bande à Loyson": Le soupçon du complot", Cahiers de Brèves, 26 (décembre 2010), p. 41-46 et 27 (juin 2011), p. 29-32. 